\title{
Strongly Singular Convolution Operators on Herz-Type Hardy Spaces with Variable Exponent
}

\author{
Hongbin Wang $\mathbb{D}^{1}$ and Dunyan Yan $\mathbb{1 D}^{2}$ \\ ${ }^{1}$ School of Mathematics and Statistics, Shandong University of Technology, Zibo 255049, China \\ ${ }^{2}$ School of Mathematical Sciences, University of Chinese Academy of Sciences, Beijing 100049, China \\ Correspondence should be addressed to Hongbin Wang; wanghb@sdut.edu.cn
}

Received 13 May 2019; Accepted 25 June 2019; Published 8 August 2019

Academic Editor: Yongsheng S. Han

Copyright (c) 2019 Hongbin Wang and Dunyan Yan. This is an open access article distributed under the Creative Commons Attribution License, which permits unrestricted use, distribution, and reproduction in any medium, provided the original work is properly cited.

We investigate the boundedness of the strongly singular convolution operators on Herz-type Hardy spaces with variable exponent.

\section{Introduction}

The theory of function spaces with variable exponents has been extensively studied by researchers since the work of Kováčik and Rákosník [1] appeared in 1991. In [2, 3] the authors defined the Herz-type Hardy spaces with variable exponent and gave some characterizations for them. In [47], the authors proved the boundedness of some integral operators on variable function spaces.

Given an open set $E \subset \mathbb{R}^{n}$ and a measurable function $p(\cdot): E \longrightarrow[1, \infty), L^{p(\cdot)}(E)$ denotes the set of measurable functions $f$ defined on $E$ such that

$$
\int_{E}\left(\frac{|f(x)|}{\lambda}\right)^{p(x)} d x<\infty
$$

holds for some $\lambda>0$.

The set $L^{p(\cdot)}(E)$ is a Banach function space when it is equipped with the Luxemburg-Nakano norm as follows:

$$
\|f\|_{L^{p(\cdot)}(E)}=\inf \left\{\lambda>0: \int_{E}\left(\frac{|f(x)|}{\lambda}\right)^{p(x)} d x \leq 1\right\} .
$$

The space is regarded as the variable $L^{p}$ space, since it generalized the standard $L^{p}$ space: if $p(x)=p$ is constant, then $L^{p(\cdot)}(E)$ is isometrically isomorphic to $L^{p}(E)$.
The space $L_{\text {loc }}^{p(\cdot)}(E)$ is defined by

$$
\begin{aligned}
& L_{\text {loc }}^{p^{(\cdot)}}(E):=\{f: f \\
& \left.\quad \in L^{p(\cdot)}(F) \text { for all compact subsets } F \subset E\right\} .
\end{aligned}
$$

Define $\mathscr{P}^{0}(E)$ to be the set of $p(\cdot): E \longrightarrow(0, \infty)$ such that

$$
\begin{aligned}
& p^{-}=\operatorname{essinf}\{p(x): x \in E\}>0, \\
& p^{+}=\operatorname{ess} \sup \{p(x): x \in E\}<\infty .
\end{aligned}
$$

Define $\mathscr{P}(E)$ to be the set of $p(\cdot): E \longrightarrow[1, \infty)$ such that

$$
p^{-}=\operatorname{essinf}\{p(x): x \in E\}>1
$$

and

$$
p^{+}=\operatorname{ess} \sup \{p(x): x \in E\}<\infty .
$$

Denote $p^{\prime}(x)=p(x) /(p(x)-1)$.

Let $f \in L_{\text {loc }}^{1}\left(\mathbb{R}^{n}\right)$. The Hardy-Littlewood maximal operator is defined by

$$
M f(x)=\sup _{r>0} \frac{1}{\left|B_{r}(x)\right|} \int_{B_{r}(x)}|f(y)| d y,
$$

where $B_{r}(x)=\left\{y \in \mathbb{R}^{n}:|x-y|<r\right\}$. Let $\mathscr{B}\left(\mathbb{R}^{n}\right)$ be the set of $p(\cdot) \in \mathscr{P}\left(\mathbb{R}^{n}\right)$ such that the Hardy-Littlewood maximal operator $M$ is bounded on $L^{p(\cdot)}\left(\mathbb{R}^{n}\right)$. 
Lemma 1 (see [8]). If $p(\cdot) \in \mathscr{P}\left(\mathbb{R}^{n}\right)$ and satisfies

$$
|p(x)-p(y)| \leq \frac{C}{-\log (|x-y|)}, \quad|x-y| \leq \frac{1}{2}
$$

and

$$
|p(x)-p(y)| \leq \frac{C}{\log (|x|+e)}, \quad|y| \geq|x|,
$$

then $(\cdot) \in \mathscr{B}\left(\mathbb{R}^{n}\right)$, that is, the Hardy-Littlewood maximal operator $M$ is bounded on $L^{p(\cdot)}\left(\mathbb{R}^{n}\right)$.

In addition, we denote the Lebesgue measure and the characteristic function of a measurable set $A \subset \mathbb{R}^{n}$ by $|A|$ and $\chi_{A}$, respectively. The notation $f \approx g$ means that there exist two constants $C_{1}, C_{2}>0$ such that $C_{1} g \leq f \leq C_{2} g$.

Next we recall the definition of the Herz spaces with variable exponent. Let $B_{k}=\left\{x \in \mathbb{R}^{n}:|x| \leq 2^{k}\right\}$ and $A_{k}=B_{k} \backslash B_{k-1}$ for $k \in \mathbb{Z}$. Denote $\mathbb{Z}_{+}$and $\mathbb{N}$ as the sets of all positive and nonnegative integers, respectively, $\chi_{k}=\chi_{A_{k}}$ for $k \in \mathbb{Z}, \widetilde{\chi}_{k}=\chi_{k}$ if $k \in \mathbb{Z}_{+}$, and $\widetilde{\chi}_{0}=\chi_{B_{0}}$.

Definition 2 (see [9]). Let $\alpha \in \mathbb{R}, 0<p \leq \infty$, and $q(\cdot) \in$ $\mathscr{P}\left(\mathbb{R}^{n}\right)$. The homogeneous Herz space with variable exponent $\dot{K}_{q(\cdot)}^{\alpha, p}\left(\mathbb{R}^{n}\right)$ is defined by

$$
\begin{aligned}
\dot{K}_{q(\cdot)}^{\alpha, p} & \left(\mathbb{R}^{n}\right) \\
\quad & \left\{f \in L_{\text {loc }}^{q(\cdot)}\left(\mathbb{R}^{n} \backslash\{0\}\right):\|f\|_{\dot{K}_{q(\cdot)}^{\alpha, p}\left(\mathbb{R}^{n}\right)}<\infty\right\},
\end{aligned}
$$

where

$$
\|f\|_{\dot{K}_{q(\cdot)}^{\alpha, p}\left(\mathbb{R}^{n}\right)}=\left\{\sum_{k=-\infty}^{\infty} 2^{k \alpha p}\left\|f \chi_{k}\right\|_{L^{q(\cdot)}\left(\mathbb{R}^{n}\right)}^{p}\right\}^{1 / p} .
$$

The nonhomogeneous Herz space with variable exponent $K_{q(\cdot)}^{\alpha, p}\left(\mathbb{R}^{n}\right)$ is defined by

$$
K_{q(\cdot)}^{\alpha, p}\left(\mathbb{R}^{n}\right)=\left\{f \in L_{\text {loc }}^{q(\cdot)}\left(\mathbb{R}^{n}\right):\|f\|_{K_{q(\cdot)}^{\alpha, p}\left(\mathbb{R}^{n}\right)}<\infty\right\},
$$

where

$$
\|f\|_{K_{q(\cdot)}^{\alpha, p}\left(\mathbb{R}^{n}\right)}=\left\{\sum_{k=0}^{\infty} 2^{k \alpha p}\left\|f \tilde{\chi}_{k}\right\|_{L^{q(\cdot)}\left(\mathbb{R}^{n}\right)}^{p}\right\}^{1 / p} .
$$

In [2], the authors gave the definition of the Herz-type Hardy space with variable exponent $H \dot{K}_{q(\cdot)}^{\alpha, p}\left(\mathbb{R}^{n}\right)$ and the atomic decomposition characterizations. $\mathcal{S}\left(\mathbb{R}^{n}\right)$ denotes the space of Schwartz functions, and $\mathcal{S}^{\prime}\left(\mathbb{R}^{n}\right)$ denotes the dual space of $\mathcal{S}\left(\mathbb{R}^{n}\right)$. Let $G_{N}(f)$ be the grand maximal function of $f$ defined by

$$
G_{N}(f)(x)=\sup _{\phi \in \mathscr{A}_{N}}\left|\phi_{\nabla}^{*}(f)(x)\right|,
$$

where

$$
\mathscr{A}_{N}=\left\{\phi \in \mathcal{S}\left(\mathbb{R}^{n}\right): \sup _{|\alpha|,|\beta| \leq N}\left|x^{\alpha} D^{\beta} \phi(x)\right| \leq 1\right\}
$$

and $N>n+1 ; \phi_{\nabla}^{*}$ is the nontangential maximal operator defined by

$$
\phi_{\nabla}^{*}(f)(x)=\sup _{|y-x|<t}\left|\phi_{t} * f(y)\right|
$$

with $\phi_{t}(x)=t^{-n} \phi(x / t)$.

Definition 3 (see [2]). Let $\alpha \in \mathbb{R}, 0<p<\infty, q(\cdot) \in \mathscr{P}\left(\mathbb{R}^{n}\right)$, and $N>n+1$.

(i) The homogeneous Herz-type Hardy space with variable exponent $H \dot{K}_{q(\cdot)}^{\alpha, p}\left(\mathbb{R}^{n}\right)$ is defined by

$$
\begin{aligned}
& H \dot{K}_{q(\cdot)}^{\alpha, p}\left(\mathbb{R}^{n}\right) \\
& \quad=\left\{f \in \mathcal{S}^{\prime}\left(\mathbb{R}^{n}\right): G_{N}(f)(x) \in \dot{K}_{q(\cdot)}^{\alpha, p}\left(\mathbb{R}^{n}\right)\right\}
\end{aligned}
$$

and

$$
\|f\|_{H \dot{K}_{q())}^{\alpha, p}\left(\mathbb{R}^{n}\right)}=\left\|G_{N}(f)\right\|_{\dot{K}_{q(\cdot)}^{\alpha, p}\left(\mathbb{R}^{n}\right)} .
$$

(ii) The nonhomogeneous Herz-type Hardy space with variable exponent $H K_{q(\cdot)}^{\alpha, p}\left(\mathbb{R}^{n}\right)$ is defined by

$$
\begin{aligned}
& H K_{q(\cdot)}^{\alpha, p}\left(\mathbb{R}^{n}\right) \\
& \quad=\left\{f \in \mathcal{S}^{\prime}\left(\mathbb{R}^{n}\right): G_{N}(f)(x) \in K_{q(\cdot)}^{\alpha, p}\left(\mathbb{R}^{n}\right)\right\}
\end{aligned}
$$

and

$$
\|f\|_{H K_{q \cdot()}^{\alpha, p}\left(\mathbb{R}^{n}\right)}=\left\|G_{N}(f)\right\|_{K_{q(\cdot)}^{\alpha, p}\left(\mathbb{R}^{n}\right)} .
$$

For $x \in \mathbb{R}$, we denote by $[x]$ the largest integer less than or equal to $x . \delta_{2}$ is the same as in Lemma 9 .

Definition 4 (see [2]). Let $n \delta_{2} \leq \alpha<\infty, q(\cdot) \in \mathscr{P}\left(\mathbb{R}^{n}\right)$, and nonnegative integer $s \geq\left[\alpha-n \delta_{2}\right]$.

(i) A function $a$ on $\mathbb{R}^{n}$ is said to be a central $(\alpha, q(\cdot))$ atom, if it satisfies

(1) supp $a \subset B(0, r)=\left\{x \in \mathbb{R}^{n}:|x|<r\right\}$

(2) $\|a\|_{L^{q(\cdot)}\left(\mathbb{R}^{n}\right)} \leq|B(0, r)|^{-\alpha / n}$

(3) $\int_{\mathbb{R}^{n}} a(x) x^{\beta} d x=0,|\beta| \leq s$

(ii) A function $a$ on $\mathbb{R}^{n}$ is said to be a central $(\alpha, q(\cdot))$ atom of restricted type, if it satisfies conditions (2), (3) and

$$
\left(1^{\prime}\right) \operatorname{supp} a \subset B(0, r), r \geq 1
$$

If $r=2^{k}$ for some $k \in \mathbb{Z}$ in Definition 4, then the corresponding central $(\alpha, q(\cdot))$-atom is called a dyadic central $(\alpha, q(\cdot))$-atom. 
Lemma 5 (see [2]). Let $n \delta_{2} \leq \alpha<\infty, 0<p<\infty$ and $q(\cdot) \in \mathscr{B}\left(\mathbb{R}^{n}\right)$. Then $f \in H \dot{K}_{q(\cdot)}^{\alpha, p}\left(\mathbb{R}^{n}\right)$ (or $\left.H K_{q(\cdot)}^{\alpha, p}\left(\mathbb{R}^{n}\right)\right)$ if and only if

$$
\begin{aligned}
& f=\sum_{k=-\infty}^{\infty} \lambda_{k} a_{k} \\
& \left(\text { or } \sum_{k=0}^{\infty} \lambda_{k} a_{k}\right),
\end{aligned}
$$

in the sense of $\mathcal{S}^{\prime}\left(\mathbb{R}^{n}\right)$,

where each $a_{k}$ is a central $(\alpha, q(\cdot))$-atom (or central $(\alpha, q(\cdot))$ atom of restricted type) with support contained in $B_{k}$ and $\sum_{k=-\infty}^{\infty}\left|\lambda_{k}\right|^{p}<\infty\left(\right.$ or $\left.\sum_{k=0}^{\infty}\left|\lambda_{k}\right|^{p}<\infty\right)$. Moreover,

$$
\begin{gathered}
\|f\|_{H \dot{K}_{q(\cdot)}^{\alpha, p}\left(\mathbb{R}^{n}\right)} \approx \inf \left(\sum_{k=-\infty}^{\infty}\left|\lambda_{k}\right|^{p}\right)^{1 / p} \\
\left(\text { or }\|f\|_{H K_{q(\cdot)}^{\alpha, p}\left(\mathbb{R}^{n}\right)} \approx \inf \left(\sum_{k=0}^{\infty}\left|\lambda_{k}\right|^{p}\right)^{1 / p}\right),
\end{gathered}
$$

where the infimum is taken over all above decomposition of $f$.

Let $\theta$ be a smooth radial cut-off function such that $\theta(\xi)=1$ if $|\xi| \geq 1$ and $\theta(\xi)=0$ if $|\xi| \leq 1 / 2$. Define the multipliers

$$
T_{b}: \widehat{T_{b} f}(\xi)=\theta(\xi) \frac{e^{i|\xi|^{b}}}{|\xi|^{n b / 2}} \widehat{f}(\xi),
$$

where $0<b<1$. The kernel for $T_{b}$ is very singular. Roughly speaking, it looks like

$$
K_{b^{\prime}}(x)=\frac{e^{i|x|^{-b^{\prime}}}}{|x|^{n}}
$$

where $b^{\prime}=b /(1-b)$. Indeed the cancellation is minimal and if one makes a quick computation for $|x| \geq 2|y|$, we have

$$
\left|K_{b^{\prime}}(x-y)-K_{b^{\prime}}(x)\right| \leq \frac{C|y|}{|x|^{n+b^{\prime}+1}} .
$$

The study of these operators in the context of $L^{q}$ spaces was carried out by Hirschman [10] and Wainger [11]. Sharp endpoint estimates were obtained by Fefferman and Stein in [12] via the duality of $H^{1}$ and BMO. Weighted $L^{q}$ norm and weak $(1,1)$ estimates were established by Chanillo in [13]. The boundedness of these operators on the weighted Herz-type Hardy spaces was proved by Xiaochun Li and Shanzhen Lu in [14].

Motivated by [2,14], we will study the boundedness of the strongly singular convolution operators $T_{b}$ on Herz-type Hardy spaces with variable exponent. The main results are as follows.

Theorem 6. Suppose that $0<p \leq 1, q(\cdot) \in \mathscr{P}\left(\mathbb{R}^{n}\right)$ satisfies conditions (8) and (9) in Lemma 1 and $\alpha=n \delta_{2}$. Then we have

$$
\left\|T_{b}(f)\right\|_{\dot{K}_{q(\cdot)}^{\alpha, p}\left(\mathbb{R}^{n}\right)} \leq C\|f\|_{H \dot{K}_{q(\cdot)}^{\alpha, p}\left(\mathbb{R}^{n}\right)},
$$

where $C$ is independent of $f$.
Theorem 7. Suppose that $0<p \leq 1, q(\cdot) \in \mathscr{P}\left(\mathbb{R}^{n}\right)$ satisfies conditions (8) and (9) in Lemma 1 and $n \delta_{2} \leq \alpha \leq n \delta_{2}+1$. Then we have

$$
\left\|T_{b}(f)\right\|_{H K_{q(\cdot)}^{\alpha, p}\left(\mathbb{R}^{n}\right)} \leq C\|f\|_{H K_{q(\cdot)}^{\alpha, p}\left(\mathbb{R}^{n}\right)},
$$

where $C$ is independent of $f$.

\section{Preliminary Lemmas}

Referring to the variable $L^{p(\cdot)}$ space, there are some important lemmas as follows.

Lemma 8 (see [1]). Let $p(\cdot) \in \mathscr{P}\left(\mathbb{R}^{n}\right)$. If $f \in L^{p(\cdot)}\left(\mathbb{R}^{n}\right)$ and $g \in L^{p^{\prime}(\cdot)}\left(\mathbb{R}^{n}\right)$, then $f g$ is integrable on $\mathbb{R}^{n}$ and

$$
\int_{\mathbb{R}^{n}}|f(x) g(x)| d x \leq r_{p}\|f\|_{L^{p(\cdot)}\left(\mathbb{R}^{n}\right)}\|g\|_{L^{p^{\prime(\cdot)}\left(\mathbb{R}^{n}\right)}},
$$

where

$$
r_{p}=1+\frac{1}{p^{-}}-\frac{1}{p^{+}} .
$$

The above inequality is named generalized Hölder's inequality with respect to the variable $L^{p}$ space.

Lemma 9 (see [9]). Let $q(\cdot) \in \mathscr{B}\left(\mathbb{R}^{n}\right)$. Then there exists a positive constant $C$ such that, for all balls $B$ in $\mathbb{R}^{n}$ and all measurable subsets $S \subset B$,

$$
\begin{aligned}
& \frac{\left\|\chi_{B}\right\|_{L^{q(\cdot)}\left(\mathbb{R}^{n}\right)}}{\left\|\chi_{S}\right\|_{L^{q(\cdot)}\left(\mathbb{R}^{n}\right)}} \leq C \frac{|B|}{|S|}, \\
& \frac{\left\|\chi_{S}\right\|_{L^{q(\cdot)}\left(\mathbb{R}^{n}\right)}}{\left\|\chi_{B}\right\|_{L^{q(\cdot)}\left(\mathbb{R}^{n}\right)}} \leq C\left(\frac{|S|}{|B|}\right)^{\delta_{1},}
\end{aligned}
$$

and

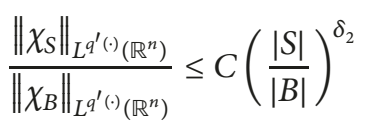

hold, where $\delta_{1}$ and $\delta_{2}$ are constants with $0<\delta_{1}, \delta_{2}<1$.

Throughout this paper $\delta_{2}$ is the same as in Lemma 9 .

Lemma 10 (see [9]). Suppose $q(\cdot) \in \mathscr{B}\left(\mathbb{R}^{n}\right)$. Then there exists a constant $C>0$ such that, for all balls $B$ in $\mathbb{R}^{n}$,

$$
\frac{1}{|B|}\left\|\chi_{B}\right\|_{L^{q(\cdot)}\left(\mathbb{R}^{n}\right)}\left\|\chi_{B}\right\|_{L^{q^{\prime}(\cdot)}\left(\mathbb{R}^{n}\right)} \leq C .
$$

Lemma 11 (see [15]). Define a variable exponent $\widetilde{q}(\cdot)$ by $1 / p(x)=1 / \widetilde{q}(x)+1 / q$ for $x \in \mathbb{R}^{n}$. Then we have

$$
\|f g\|_{L^{p \cdot(\cdot)}\left(\mathbb{R}^{n}\right)} \leq C\|f\|_{L^{\tilde{q} \cdot(\cdot)}\left(\mathbb{R}^{n}\right)}\|g\|_{L^{q}\left(\mathbb{R}^{n}\right)},
$$

for all measurable functions $f$ and $g$. 
Lemma 12 (see [16]). Let $p(\cdot) \in \mathscr{P}\left(\mathbb{R}^{n}\right)$ satisfy conditions (8) and (9) in Lemma 1. Then

$$
\left\|\chi_{Q}\right\|_{L^{p(\cdot)}\left(\mathbb{R}^{n}\right)} \approx \begin{cases}|Q|^{1 / p(x)} & \text { if }|Q| \leq 2^{n} \text { and } x \in Q, \\ |Q|^{1 / p(\infty)} & \text { if }|Q| \geq 1\end{cases}
$$

for every cube (or ball) $Q \subset \mathbb{R}^{n}$, where $p(\infty)=\lim _{x \rightarrow \infty} p(x)$.

A nonnegative locally integrable function $\omega(x)$ on $\mathbb{R}^{n}$ is said to belong to $A_{p}(1<p<\infty)$, if there is a constant $C>0$ such that

$$
\begin{aligned}
& \sup _{Q}\left(\frac{1}{|Q|} \int_{Q} \omega(x) d x\right)\left(\frac{1}{|Q|} \int_{Q} \omega(x)^{1-p^{\prime}} d x\right)^{p-1} \\
& \quad \leq C<\infty
\end{aligned}
$$

where $p^{\prime}=p /(p-1) ; Q$ denotes a cube in $\mathbb{R}^{n}$ with its sides parallel to the coordinate axes.

The weighted $\left(L^{p}, L^{p}\right)$ boundedness of $T_{b}$ has been proved by Chanillo [13].

Lemma 13 (see [13]). Let $\omega \in A_{p}, 1<p<\infty$. Then

$$
\int_{\mathbb{R}^{n}}\left|T_{b}(f)(x)\right|^{p} \omega(x) d x \leq C \int_{\mathbb{R}^{n}}|f(x)|^{p} \omega(x) d x .
$$

Lemma 14 (see [5]). Given a family $\mathscr{F}$ and an open set $E \subset$ $\mathbb{R}^{n}$, assume that for some $p_{0}, 0<p_{0}<\infty$ and for every $\omega \in$ $A_{\infty}$,

$$
\int_{E} f(x)^{p_{0}} \omega(x) d x \leq C_{0} \int_{E} g(x)^{p_{0}} \omega(x) d x,
$$

$$
(f, g) \in \mathscr{F} .
$$

Given $p(\cdot) \in \mathscr{P}^{0}(E)$ such that $p(\cdot)$ satisfies (8) and (9) in Lemma 1 , then for all $(f, g) \in \mathscr{F}$ such that $f \in L^{p(\cdot)}(E)$

$$
\|f\|_{L^{p^{(\cdot)}(E)}} \leq C\|g\|_{L^{p^{(\cdot)}(E)}} .
$$

Since $A_{p} \subset A_{\infty}$, by Lemmas 13 and 14 it is easy to get the $\left(L^{p(\cdot)}\left(\mathbb{R}^{n}\right), L^{p(\cdot)}\left(\mathbb{R}^{n}\right)\right)$-boundedness of the strongly singular convolution operators $T_{b}$.

To prove our main results, we also need the following lemmas.

Lemma 15 (see [11]). The kernel for the multiplier operator $T_{b}(f)(x)$ is given by

$$
C \frac{e^{i \alpha_{b}|x|^{-b^{\prime}}}}{|x|^{n}} \chi(|x| \leq 1)+h(x), \quad b^{\prime}=\frac{b}{(1-b)},
$$

with $|h(x)| \leq C(1+|x|)^{-(n+1)}+C|x|^{-n+\varepsilon} \chi(|x| \leq 1), \varepsilon>0$. Here $\alpha_{b}=b^{b /(1-b)}-b^{1 /(1-b)}$ and $\varepsilon$ depend only on $b$.

Lemma 16 (see [13]). Let $\widetilde{K}_{b^{\prime}, s}(x)=e^{i \alpha_{b}|x|^{-b^{\prime}}} /|x|^{n\left(b^{\prime}+2\right) / s}$ and $\left(b^{\prime}+2\right) / s<1$. Then

$$
\left\|\widetilde{K}_{b^{\prime}, s} * f\right\|_{s} \leq C\|f\|_{s^{\prime}}, \quad \frac{1}{s}+\frac{1}{s^{\prime}}=1 .
$$

\section{The Proof of Main Results}

Firstly we give the proof of Theorem 6.

Proof of Theorem 6. Let $f \in H \dot{K}_{q(\cdot)}^{\alpha, p}\left(\mathbb{R}^{n}\right)$. By Lemma 5, we have

$$
f(x)=\sum_{j=-\infty}^{\infty} \lambda_{j} a_{j}
$$

where

$$
\|f\|_{H \dot{K}_{q(\cdot)}^{\alpha, p}\left(\mathbb{R}^{n}\right)} \approx \inf \left(\sum_{j=-\infty}^{\infty}\left|\lambda_{j}\right|^{p}\right)^{1 / p},
$$

the infimum is taken over the above decomposition of $f$, and $a_{j}$ is a dyadic central $(\alpha, q(\cdot))$-atom with the support $B_{j}$. Then we have

$$
\begin{aligned}
& \left\|T_{b}(f)\right\|_{\dot{K}_{q(\cdot)}^{\alpha, p}\left(\mathbb{R}^{n}\right)}^{p}=\sum_{k=-\infty}^{\infty} 2^{k \alpha p}\left\|T_{b}(f) \chi_{k}\right\|_{L^{q(\cdot)}\left(\mathbb{R}^{n}\right)}^{p} \\
& \leq C \sum_{k=-\infty}^{\infty} 2^{k \alpha p}\left(\sum_{j=-\infty}^{k-2}\left|\lambda_{j}\right|\left\|T_{b}\left(a_{j}\right) \chi_{k}\right\|_{L^{q(\cdot)}\left(\mathbb{R}^{n}\right)}\right)^{p} \\
& \quad+C \sum_{k=-\infty}^{\infty} 2^{k \alpha p}\left(\sum_{j=k-1}^{\infty}\left|\lambda_{j}\right|\left\|T_{b}\left(a_{j}\right) \chi_{k}\right\|_{L^{q(\cdot)}\left(\mathbb{R}^{n}\right)}\right)^{p} \\
& =: C I_{1}+C I_{2} .
\end{aligned}
$$

We first estimate $I_{2}$; by $0<p \leq 1$ and the $\left(L^{q(\cdot)}\left(\mathbb{R}^{n}\right), L^{q(\cdot)}\left(\mathbb{R}^{n}\right)\right)$-boundedness of $T_{b}$ we have

$$
\begin{aligned}
I_{2} & \leq C \sum_{k=-\infty}^{\infty} 2^{k \alpha p}\left(\sum_{j=k-1}^{\infty}\left|\lambda_{j}\right|\left\|a_{j}\right\|_{L^{q(\cdot)}\left(\mathbb{R}^{n}\right)}\right)^{p} \\
& \leq C \sum_{k=-\infty}^{\infty}\left(\sum_{j=k-1}^{\infty}\left|\lambda_{j}\right| 2^{(k-j) \alpha}\right)^{p} \\
& \leq C \sum_{j=-\infty}^{\infty}\left|\lambda_{j}\right|^{p}\left(\sum_{k=-\infty}^{j+1} 2^{(k-j) \alpha p}\right) \leq C \sum_{j=-\infty}^{\infty}\left|\lambda_{j}\right|^{p} \\
& \leq C\|f\|_{H \dot{K}_{q(\cdot)}^{\alpha, p}\left(\mathbb{R}^{n}\right)} \cdot
\end{aligned}
$$

Now we estimate $I_{1}$. Let

$$
K_{b^{\prime}}(x)=C \frac{i \alpha_{b}|x|^{-b^{\prime}}}{|x|^{n}} \chi(|x| \leq 1) .
$$

By Lemma 15 and the Minkowski inequality, we have

$$
\begin{aligned}
I_{1} \leq & \sum_{k=-\infty}^{\infty} 2^{k \alpha p}\left(\sum_{j=-\infty}^{k-2}\left|\lambda_{j}\right|\left\|\left(K_{b^{\prime}} * a_{j}\right) \chi_{k}\right\|_{L^{q(\cdot)}\left(\mathbb{R}^{n}\right)}\right)^{p} \\
& +\sum_{k=-\infty}^{\infty} 2^{k \alpha p}\left(\sum_{j=-\infty}^{k-2}\left|\lambda_{j}\right|\left\|\left(h * a_{j}\right) \chi_{k}\right\|_{L^{q(\cdot)}\left(\mathbb{R}^{n}\right)}\right)^{p} \\
= & I_{11}+I_{12} .
\end{aligned}
$$


To estimate the term $I_{12}$, we need the pointwise estimate for $h * a_{j}(x)$.

Let $|x| \geq 2^{j}$. By generalized Hölder's inequality we have

$$
\begin{array}{rl}
\mid h & * a_{j}(x)\left|\leq \int_{|t| \leq r}\right| h(x-t)|| a_{j}(t) \mid d t \\
& \leq C \int_{|t| \leq r}\left|a_{j}(t)\right| \\
& \cdot\left[\frac{1}{(1+|x-t|)^{n+1}}+\frac{\chi(|x-t| \leq 1)}{|x-t|^{n-\varepsilon}}\right] d t \\
& \leq C\left(\int_{|t| \leq r}\left|a_{j}(t)\right| d t\right) \\
& \cdot\left[\frac{1}{(1+|x|)^{n+1}}+\frac{\chi(|x| \leq 2)}{|x|^{n-\varepsilon}}\right] \leq C\left\|a_{j}\right\|_{L^{q(\cdot)}\left(\mathbb{R}^{n}\right)} \\
& \cdot\left\|\chi_{j}\right\|_{L^{q^{\prime}(\cdot)}\left(\mathbb{R}^{n}\right)}\left[\frac{1}{(1+|x|)^{n+1}}+\frac{\chi(|x| \leq 2)}{|x|^{n-\varepsilon}}\right] .
\end{array}
$$

Therefore, by $n \delta_{2}=\alpha, 0<p \leq 1$, Lemmas 9 and 10, the Minkowski inequality, and generalized Hölder's inequality we have

$$
\begin{aligned}
& I_{12} \leq C \sum_{k=-\infty}^{\infty} 2^{k \alpha p}\left(\sum_{j=-\infty}^{k-2}\left|\lambda_{j}\right|\left\|a_{j}\right\|_{L^{q(\cdot)}\left(\mathbb{R}^{n}\right)}\left\|\chi_{j}\right\|_{L^{q^{\prime}(\cdot)}\left(\mathbb{R}^{n}\right)}\right. \\
& \left.\cdot\left\|\frac{1}{(1+|\cdot|)^{n+1}} \chi_{k}(\cdot)\right\|_{L^{q(\cdot)}\left(\mathbb{R}^{n}\right)}\right)^{p}+C \sum_{k=-\infty}^{\infty} 2^{k \alpha p} \\
& \cdot\left(\sum_{j=-\infty}^{k-2}\left|\lambda_{j}\right|\left\|a_{j}\right\|_{L^{q^{(\cdot)}\left(\mathbb{R}^{n}\right)}}\left\|\chi_{j}\right\|_{L^{q^{(\cdot)}\left(\mathbb{R}^{n}\right)}} \| \frac{\chi(|\cdot| \leq 2)}{|\cdot|^{n-\varepsilon}}\right. \\
& \left.\cdot \chi_{k}(\cdot) \|_{L^{(\cdot)}\left(\mathbb{R}^{n}\right)}\right)^{p} \leq C \sum_{k=-\infty}^{\infty}\left(\sum_{j=-\infty}^{k-2}\left|\lambda_{j}\right| 2^{k \alpha}\right. \\
& \cdot \frac{1}{\left(1+2^{k}\right)^{n+1}}\left\|a_{j}\right\|_{L^{q(\cdot)}\left(\mathbb{R}^{n}\right)}\left\|\chi_{B_{j}}\right\|_{L^{q^{(\cdot)}\left(\mathbb{R}^{n}\right)}} \\
& \left.\cdot\left\|\chi_{B_{k}}\right\|_{L^{q(\cdot)}\left(\mathbb{R}^{n}\right)}\right)^{p}+C \sum_{k=-\infty}^{1}\left(\sum_{j=-\infty}^{k-2}\left|\lambda_{j}\right| 2^{k \alpha} \frac{1}{2^{k(n-\varepsilon)}}\right. \\
& \left.\cdot\left\|a_{j}\right\|_{L^{q(\cdot)}\left(\mathbb{R}^{n}\right)}\left\|\chi_{B_{j}}\right\|_{L^{q^{(\cdot)}\left(\mathbb{R}^{n}\right)}}\left\|\chi_{B_{k}}\right\|_{L^{q^{(\cdot)}\left(\mathbb{R}^{n}\right)}}\right)^{p} \\
& \leq C \sum_{k=-\infty}^{\infty}\left(\sum_{j=-\infty}^{k-2}\left|\lambda_{j}\right| \frac{2^{k \alpha+k n-j \alpha}}{\left(1+2^{k}\right)^{n+1}} \frac{\left\|\chi_{B_{j}}\right\|_{L^{q^{\prime}(\cdot)\left(\mathbb{R}^{n}\right)}}}{\left\|\chi_{B_{k}}\right\|_{L^{q^{\prime}(\cdot)\left(\mathbb{R}^{n}\right)}}}\right)^{p}
\end{aligned}
$$

$$
\begin{aligned}
& +C \sum_{k=-\infty}^{1}\left(\sum_{j=-\infty}^{k-2}\left|\lambda_{j}\right| \frac{2^{k \alpha+k n-j \alpha}}{2^{k(n-\varepsilon)}} \frac{\left\|\chi_{B_{j}}\right\|_{L^{q^{\prime}(\cdot)\left(\mathbb{R}^{n}\right)}}}{\left\|\chi_{B_{k}}\right\|_{L^{q^{\prime}(\cdot)\left(\mathbb{R}^{n}\right)}}}\right)^{p} \\
& \leq C \sum_{k=-\infty}^{\infty}\left(\sum_{j=-\infty}^{k-2}\left|\lambda_{j}\right| \frac{2^{k n+(j-k)\left(n \delta_{2}-\alpha\right)}}{\left(1+2^{k}\right)^{n+1}}\right)^{p} \\
& +C \sum_{k=-\infty}^{1}\left(\sum_{j=-\infty}^{k-2}\left|\lambda_{j}\right| \frac{2^{k n+(j-k)\left(n \delta_{2}-\alpha\right)}}{2^{k(n-\varepsilon)}}\right)^{p} \\
& \leq C \sum_{k=-\infty}^{\infty}\left(\sum_{j=-\infty}^{k-2}\left|\lambda_{j}\right| \frac{2^{k n}}{\left(1+2^{k}\right)^{n+1}}\right)^{p} \\
& +C \sum_{k=-\infty}^{1}\left(\sum_{j=-\infty}^{k-2}\left|\lambda_{j}\right| \frac{2^{k n}}{2^{k(n-\varepsilon)}}\right)^{p} \leq C \sum_{j=-\infty}^{\infty}\left|\lambda_{j}\right|^{p} \\
& \cdot\left(\sum_{k=j+2}^{\infty} \frac{2^{k n p}}{\left(1+2^{k}\right)^{(n+1) p}}\right)+C \sum_{j=-\infty}^{-1}\left|\lambda_{j}\right|^{p} \\
& \cdot\left(\sum_{k=j+2}^{1} \frac{2^{k n p}}{2^{k(n-\varepsilon) p}}\right) \leq C \sum_{j=-\infty}^{\infty}\left|\lambda_{j}\right|^{p}\left(\sum_{k=j+2}^{1} 2^{k n p}\right. \\
& \left.+\sum_{k=1}^{\infty} \frac{1}{2^{k p}}\right)+C \sum_{j=-\infty}^{-1}\left|\lambda_{j}\right|^{p}\left(\sum_{k=j+2}^{1} 2^{k \varepsilon p}\right) \\
& \leq C \sum_{j=-\infty}^{\infty}\left|\lambda_{j}\right|^{p} \text {. }
\end{aligned}
$$

What remains is estimating $I_{11}$. Let $2^{j_{0}-1}<2^{j(1-b)} \leq 2^{j_{0}}$ for some $j_{0} \in \mathbb{Z}$, where $b$ is the same as the above. Then it follows that

$$
\begin{aligned}
& I_{11} \\
& =\sum_{k=-\infty}^{j_{0}} 2^{k \alpha p}\left(\sum_{j=-\infty}^{k-2}\left|\lambda_{j}\right|\left\|\left(K_{b^{\prime}} * a_{j}\right) \chi_{k}\right\|_{L^{q(\cdot)}\left(\mathbb{R}^{n}\right)}\right)^{p} \\
& +\sum_{k=j_{0}+1}^{\infty} 2^{k \alpha p}\left(\sum_{j=-\infty}^{k-2}\left|\lambda_{j}\right|\left\|\left(K_{b^{\prime}} * a_{j}\right) \chi_{k}\right\|_{L^{q(\cdot)}\left(\mathbb{R}^{n}\right)}\right)^{p} \\
& =I_{13}+I_{14} \text {. }
\end{aligned}
$$

To estimate the term $I_{14}$, we need the pointwise estimate for $K_{b^{\prime}} * a_{j}(x)$. Let $|x| \geq 2^{j}$. Then, by the vanishing moment condition on $a_{j}(x)$, we have

$$
\left|K_{b^{\prime}} * a_{j}(x)\right| \leq \int_{B_{j}}\left|K_{b^{\prime}}(x-y)-K_{b^{\prime}}(x)\right|\left|a_{j}(y)\right| d y .
$$


From the condition of $K_{b^{\prime}}(x),\left|K_{b^{\prime}}(x-y)-K_{b^{\prime}}(x)\right| \leq$ $C\left(|y| /|x|^{n+b^{\prime}+1}\right)$, if $|x| \geq 2|y|$, it follows that

$$
\begin{aligned}
\left|K_{b^{\prime}} * a_{j}(x)\right| & \leq \frac{C 2^{j}}{|x|^{n+b^{\prime}+1}} \int_{B_{j}}\left|a_{j}(y)\right| d y \\
& \leq \frac{C 2^{j}}{|x|^{n+b^{\prime}+1}}\left\|a_{j}\right\|_{L^{q(\cdot)}\left(\mathbb{R}^{n}\right)}\left\|\chi_{B_{j}}\right\|_{L^{q^{\prime}(\cdot)\left(\mathbb{R}^{n}\right)}} .
\end{aligned}
$$

Note that $b^{\prime}=b /(1-b)$; that is, $(1-b)\left(b^{\prime}+1\right)=1$. Since $n \delta_{2}=\alpha, 0<p \leq 1$, and $2^{j_{0}-1}<2^{j(1-b)} \leq 2^{j_{0}}$, then by Lemmas 9 and 10 we have

$$
\begin{aligned}
& I_{14} \leq C \sum_{k=j_{0}+1}^{\infty} 2^{k \alpha p}\left(\sum_{j=-\infty}^{k-2}\left|\lambda_{j}\right| \frac{2^{j}}{2^{k\left(n+b^{\prime}+1\right)}}\left\|a_{j}\right\|_{L^{q(\cdot)}\left(\mathbb{R}^{n}\right)}\right. \\
& \left.\cdot\left\|\chi_{B_{j}}\right\|_{L^{q^{\prime} \cdot(\cdot)\left(\mathbb{R}^{n}\right)}}\left\|\chi_{k}\right\|_{L^{q(\cdot)}\left(\mathbb{R}^{n}\right)}\right)^{p} \\
& \leq C \sum_{k=j_{0}+1}^{\infty}\left(\sum_{j=-\infty}^{k-2}\left|\lambda_{j}\right| \frac{2^{k \alpha-j \alpha} 2^{j} 2^{k n}}{2^{k\left(n+b^{\prime}+1\right)}}\right. \\
& \left.\cdot \frac{\left\|\chi_{B_{j}}\right\|_{L^{q^{\prime}(\cdot)\left(\mathbb{R}^{n}\right)}}}{\left\|\chi_{B_{k}}\right\|_{L^{q^{\prime}(\cdot)\left(\mathbb{R}^{n}\right)}}}\right)^{p} \leq C \sum_{j=-\infty}^{\infty}\left|\lambda_{j}\right|^{p} \sum_{k=j_{0}+1}^{\infty} \frac{2^{j p}}{2^{k\left(b^{\prime}+1\right) p}} \\
& \leq C \sum_{j=-\infty}^{\infty}\left|\lambda_{j}\right|^{p} \frac{2^{j p}}{2^{j_{0}\left(b^{\prime}+1\right) p}} \leq C \sum_{j=-\infty}^{\infty}\left|\lambda_{j}\right|^{p} \\
& \cdot \frac{2^{j p}}{2^{j(1-b)\left(b^{\prime}+1\right) p}} \leq C \sum_{j=-\infty}^{\infty}\left|\lambda_{j}\right|^{p} .
\end{aligned}
$$

Now to estimate $I_{13}$, we split $K_{b^{\prime}} * a_{j}(x)$ as follows:

$$
\begin{gathered}
K_{b^{\prime}} * a_{j}(x)=C \int_{B_{j}} \frac{e^{i \alpha_{b}|x-y|^{-b^{\prime}}}}{|x-y|^{n\left(b^{\prime}+2\right) / s}} \\
\cdot\left[\frac{1}{|x-y|^{n\left(1-\left(b^{\prime}+2\right) / s\right)}}-\frac{1}{|x|^{n\left(1-\left(b^{\prime}+2\right) / s\right)}}\right] a_{j}(y) d y \\
+C\left(\widetilde{K}_{b^{\prime}, s} * a_{j}(x)\right) \frac{1}{|x|^{n\left(1-\left(b^{\prime}+2\right) / s\right)}}=: E(x)+F(x),
\end{gathered}
$$

where $\widetilde{K}_{b^{\prime}, s}$ is the same as in Lemma 16 and let $s>\max \left\{q^{+}, 2\right\}$ satisfy $\left(b^{\prime}+2\right) / s<1$.

Applying the mean value theorem to the term brackets in the integrand of $E(x)$, then for $|x| \geq 2^{j}$ we have the pointwise estimate for $E(x)$ as follows:

$$
\begin{aligned}
|E(x)| & \leq C \int_{B_{j}} \frac{|y|}{|x|^{n+1}}\left|a_{j}(y)\right| d y \\
& \leq C \frac{2^{j}}{|x|^{n+1}}\left\|a_{j}\right\|_{L^{q(\cdot)}\left(\mathbb{R}^{n}\right)}\left\|\chi_{B_{j}}\right\|_{L^{q^{\prime}(\cdot)}\left(\mathbb{R}^{n}\right)} .
\end{aligned}
$$

On the other hand, since $0<p \leq 1$, by the Minkowski inequality we get

$$
\begin{aligned}
I_{13} \leq & \sum_{k=-\infty}^{j_{0}} 2^{k \alpha p} \sum_{j=-\infty}^{k-2}\left|\lambda_{j}\right|^{p}\left\|E \chi_{k}\right\|_{L^{q(\cdot)}\left(\mathbb{R}^{n}\right)}^{p} \\
& +\sum_{k=-\infty}^{j_{0}} 2^{k \alpha p} \sum_{j=-\infty}^{k-2}\left|\lambda_{j}\right|^{p}\left\|F \chi_{k}\right\|_{L^{q(\cdot)}\left(\mathbb{R}^{n}\right)}^{p}=: I_{15}+I_{16} .
\end{aligned}
$$

For $I_{15}$, using $n \delta_{2}=\alpha$, the pointwise estimate for $E(x)$, and Lemmas 9 and 10 we have

$$
\begin{aligned}
& I_{15} \leq C \sum_{k=-\infty}^{j_{0}} 2^{k \alpha p} \sum_{j=-\infty}^{k-2}\left|\lambda_{j}\right|^{p} \frac{2^{j p}}{2^{k(n+1) p}}\left\|a_{j}\right\|_{L^{q(\cdot)}\left(\mathbb{R}^{n}\right)}^{p} \\
& \cdot\left\|\chi_{B_{j}}\right\|_{L^{q^{(\cdot)}\left(\mathbb{R}^{n}\right)}}^{p}\left\|\chi_{k}\right\|_{L^{q(\cdot)}\left(\mathbb{R}^{n}\right)}^{p} \leq C \sum_{j=-\infty}^{\infty}\left|\lambda_{j}\right|^{p} \\
& \cdot \sum_{k=j+2}^{j_{0}} \frac{2^{j p}}{2^{k(n+1) p}} 2^{(k-j) \alpha p}\left(2^{k n} \frac{\left\|\chi_{B_{j}}\right\|_{L^{q^{\prime} \cdot(\cdot)}\left(\mathbb{R}^{n}\right)}}{\left\|\chi_{B_{k}}\right\|_{L^{q^{\prime} \cdot(\cdot)}\left(\mathbb{R}^{n}\right)}}\right)^{p} \\
& \quad \leq C \sum_{j=-\infty}^{\infty}\left|\lambda_{j}\right|^{p} .
\end{aligned}
$$

Finally, we estimate $I_{16}$. Noting that $x \in A_{k}$, we get

$$
\begin{aligned}
& I_{16}=\sum_{k=-\infty}^{j_{0}} 2^{k \alpha p} \sum_{j=-\infty}^{k-2}\left|\lambda_{j}\right|^{p}\left\|F \chi_{k}\right\|_{L^{q(\cdot)}\left(\mathbb{R}^{n}\right)}^{p} \leq C \sum_{k=-\infty}^{j_{0}} 2^{k \alpha p} \\
& \cdot \sum_{j=-\infty}^{k-2}\left|\lambda_{j}\right|^{p} \\
& \cdot\left\|\left(\widetilde{K}_{b^{\prime}, s} * a_{j}(\cdot)\right) \frac{1}{|\cdot|^{n\left(1-\left(b^{\prime}+2\right) / s\right)}} \chi_{k}(\cdot)\right\|_{L^{q(\cdot)}\left(\mathbb{R}^{n}\right)}^{p} \\
& \leq C \sum_{k=-\infty}^{j_{0}} 2^{k \alpha p} \sum_{j=-\infty}^{k-2}\left|\lambda_{j}\right|^{p}
\end{aligned}
$$

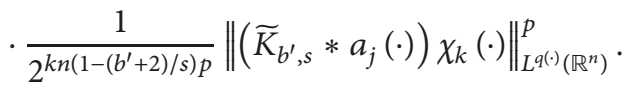

Noting $s>\max \left\{q^{+}, 2\right\}, s^{\prime}<\min \left\{q^{-}, 2\right\}, 1 / s+1 / s^{\prime}=1$, we denote $\widetilde{q}(\cdot)>s /(s-2)$ and $1 / q(x)=1 / \widetilde{q}(x)+1 / s$.

When $\left|B_{k}\right| \leq 2^{n}$ and $x_{k} \in B_{k}$, by Lemma 12 we have

$$
\left\|\chi_{B_{k}}\right\|_{L^{\tilde{q}^{(\cdot)}\left(\mathbb{R}^{n}\right)}} \approx\left|B_{k}\right|^{1 / \widetilde{q}\left(x_{k}\right)} \approx\left\|\chi_{B_{k}}\right\|_{L^{q(\cdot)}\left(\mathbb{R}^{n}\right)}\left|B_{k}\right|^{-1 / s} .
$$

When $\left|B_{k}\right| \geq 1$ we have

$$
\left\|\chi_{B_{k}}\right\|_{L^{\tilde{q}(\cdot)}\left(\mathbb{R}^{n}\right)} \approx\left|B_{k}\right|^{1 / \widetilde{q}(\infty)} \approx\left\|\chi_{B_{k}}\right\|_{L^{q(\cdot)}\left(\mathbb{R}^{n}\right)}\left|B_{k}\right|^{-1 / s} .
$$

So we obtain

$$
\left\|\chi_{B_{k}}\right\|_{L^{\tilde{q} \cdot(\cdot)\left(\mathbb{R}^{n}\right)}} \approx\left\|\chi_{B_{k}}\right\|_{L^{q(\cdot)}\left(\mathbb{R}^{n}\right)}\left|B_{k}\right|^{-1 / s} .
$$


In similar method we can obtain

$$
\left\|\chi_{B_{j}}\right\|_{L^{q(\cdot) /\left(q(\cdot)-s^{\prime}\right)\left(\mathbb{R}^{n}\right)}} \approx\left|B_{j}\right|^{\left.(q \cdot \cdot)-s^{\prime}\right) / q(\cdot)}
$$

and

$$
\left\|\chi_{B_{j}}\right\|_{L^{q^{\prime}(\cdot)\left(\mathbb{R}^{n}\right)}} \approx\left|B_{j}\right|^{1 / q^{\prime}(\cdot)} .
$$

Thus by Lemmas 11,12 , and 16 we have

$$
\begin{aligned}
& \left\|\left(\widetilde{K}_{b^{\prime}, s} * a_{j}(\cdot)\right) \chi_{k}(\cdot)\right\|_{L^{q(\cdot)}\left(\mathbb{R}^{n}\right)} \\
& \leq C\left\|\left(\widetilde{K}_{b^{\prime}, s} * a_{j}(\cdot)\right) \chi_{k}(\cdot)\right\|_{L^{s}\left(\mathbb{R}^{n}\right)}\left\|\chi_{k}\right\|_{L^{\bar{q} \cdot(\cdot)}\left(\mathbb{R}^{n}\right)} \\
& \leq C\left\|a_{j}\right\|_{L^{s^{\prime}\left(\mathbb{R}^{n}\right)}}\left\|\chi_{B_{k}}\right\|_{L^{\bar{q}(\cdot)}\left(\mathbb{R}^{n}\right)} \leq C\left\|a_{j}\right\|_{L^{s^{\prime}\left(\mathbb{R}^{n}\right)}} \\
& \cdot\left\|\chi_{B_{k}}\right\|_{L^{q(\cdot)}\left(\mathbb{R}^{n}\right)}\left|B_{k}\right|^{-1 / s} \leq C\left\|\left|a_{j}\right|^{s^{\prime}}\right\|_{L^{q(\cdot) / s^{\prime}}\left(\mathbb{R}^{n}\right)}^{1 / s^{\prime}} \\
& \cdot\left\|\chi_{B_{j}}\right\|_{L^{(\cdot) /\left(q(\cdot)-s^{\prime}\right)}\left(\mathbb{R}^{n}\right)}^{1 / s^{\prime}}\left\|\chi_{B_{k}}\right\|_{L^{q(\cdot)}\left(\mathbb{R}^{n}\right)}\left|B_{k}\right|^{-1 / s} \\
& \leq C\left\|a_{j}\right\|_{L^{q(\cdot)}\left(\mathbb{R}^{n}\right)}\left|B_{j}\right|^{\left.\left((q(\cdot)) s^{\prime}\right) / q(\cdot)\right)\left(1 / s^{\prime}\right)}\left\|\chi_{B_{k}}\right\|_{L^{q^{(\cdot)}\left(\mathbb{R}^{n}\right)}} \\
& \cdot\left|B_{k}\right|^{-1 / s}=C\left\|a_{j}\right\|_{L^{q \cdot(\cdot)\left(\mathbb{R}^{n}\right)}}\left|B_{j}\right|^{1-1 / q(\cdot)}\left|B_{j}\right|^{-1 / s} \\
& \cdot\left\|\chi_{B_{k}}\right\|_{L^{q(\cdot)}\left(\mathbb{R}^{n}\right)}\left|B_{k}\right|^{-1 / s} \leq C\left\|a_{j}\right\|_{L^{q(\cdot)}\left(\mathbb{R}^{n}\right)}\left\|\chi_{B_{j}}\right\|_{L^{q^{\prime} \cdot(\cdot)}\left(\mathbb{R}^{n}\right)} \\
& \cdot\left|B_{j}\right|^{-1 / s}\left\|\chi_{B_{k}}\right\|_{L^{q^{(\cdot)}\left(\mathbb{R}^{n}\right)}}\left|B_{k}\right|^{-1 / s} .
\end{aligned}
$$

So by using $0<p \leq 1, n \delta_{2}=\alpha, 2^{j_{0}-1}<2^{j(1-b)} \leq 2^{j_{0}},(1-$ $b)\left(b^{\prime}+1\right)=1$, and Lemmas 9 and 10 we have

$$
\begin{aligned}
& I_{16} \leq C \sum_{k=-\infty}^{j_{0}} 2^{k \alpha p} \sum_{j=-\infty}^{k-2}\left|\lambda_{j}\right|^{p} \frac{1}{2^{k n\left(1-\left(b^{\prime}+2\right) / s\right) p}} \\
& \times\left(\left\|a_{j}\right\|_{L^{q(\cdot)}\left(\mathbb{R}^{n}\right)}\left\|\chi_{B_{j}}\right\|_{L^{q^{\prime}(\cdot)\left(\mathbb{R}^{n}\right)}}\left|B_{j}\right|^{-1 / s}\left\|\chi_{B_{k}}\right\|_{L^{q(\cdot)}\left(\mathbb{R}^{n}\right)}\right. \\
& \left.\cdot\left|B_{k}\right|^{-1 / s}\right)^{p} \leq C \sum_{k=-\infty}^{j_{0}} \sum_{j=-\infty}^{k-2}\left|\lambda_{j}\right|^{p} 2^{(k-j) \alpha p} 2^{k n p\left(b^{\prime}+1\right) / s} \\
& \cdot\left(\frac{\left\|\chi_{B_{j}}\right\|_{L^{q^{(\cdot)}\left(\mathbb{R}^{n}\right)}}}{\left\|\chi_{B_{k}}\right\|_{L^{q^{\prime}(\cdot)\left(\mathbb{R}^{n}\right)}}}\right)^{p}\left|B_{j}\right|^{-p / s} \leq C \sum_{j=-\infty}^{\infty}\left|\lambda_{j}\right|^{p} \\
& \cdot \sum_{k=j+2}^{j_{0}} 2^{k n p\left(b^{\prime}+1\right) / s}\left|B_{j}\right|^{-p / s} \leq C \sum_{j=-\infty}^{\infty}\left|\lambda_{j}\right|^{p} \\
& \cdot \frac{2^{j_{0} n p\left(b^{\prime}+1\right) / s}}{2^{j n p / s}} \leq C \sum_{j=-\infty}^{\infty}\left|\lambda_{j}\right|^{p} \frac{2^{j n p(1-b)\left(b^{\prime}+1\right) / s}}{2^{j n p / s}} \\
& \leq C \sum_{j=-\infty}^{\infty}\left|\lambda_{j}\right|^{p} .
\end{aligned}
$$


8

Journal of Function Spaces

Suppose that $y, t$ satisfy $|x-y|<t$. Let $|x|>2^{j+2}$ and $\phi \epsilon$ $\mathscr{K}_{m}$, where $m \in \mathbb{N}$ and $\mathscr{K}_{m}=\left\{\phi \in \mathcal{S}\left(\mathbb{R}^{n}\right): \sup _{u \in \mathbb{R}^{n},|\alpha| \leq m}(1+\right.$ $\left.|u|)^{m+n}\left|D^{\alpha} \phi(u)\right| \leq 1\right\}$. By the vanishing moment condition on $a_{j}(x)$, it is easy to prove that $\int_{\mathbb{R}^{n}} T_{b} a_{j}(x) d x=0$. So we have

$$
\begin{aligned}
& \left|\left(T_{b} a_{j} * \phi_{t}\right)(y)\right| \\
& =\left|\int_{\mathbb{R}^{n}} t^{-n} T_{b} a_{j}(z)\left(\phi\left(\frac{y-z}{t}\right)-\phi\left(\frac{y}{t}\right)\right) d z\right| \\
& \leq\left|\int_{|z| \leq 2^{j+1}} t^{-n} T_{b} a_{j}(z)\left(\phi\left(\frac{y-z}{t}\right)-\phi\left(\frac{y}{t}\right)\right) d z\right| \\
& \quad+\left|\int_{|z|>2^{j+1}} t^{-n} T_{b} a_{j}(z)\left(\phi\left(\frac{y-z}{t}\right)-\phi\left(\frac{y}{t}\right)\right) d z\right| \\
& =: J_{11}+J_{12} .
\end{aligned}
$$

For $J_{11}$, by Lemma 9, the generalized Hölder inequality, and the mean value theorem, we obtain

$$
\begin{aligned}
& J_{11} \leq C\left\|T_{b} a_{j}\right\|_{L^{q(\cdot)}\left(\mathbb{R}^{n}\right)} \\
& \cdot t^{-n}\left\|\left(\phi\left(\frac{y-\cdot}{t}\right)-\phi\left(\frac{y}{t}\right)\right) \chi_{B_{j+1}}\right\|_{L^{q^{\prime}(\cdot)}\left(\mathbb{R}^{n}\right)} \\
& \leq C\left\|a_{j}\right\|_{L^{q \cdot \cdot\left(\mathbb{R}^{n}\right)}} \\
& \cdot t^{-n}\left\|\sum_{|\beta|=1}\left|D^{\beta} \phi\left(\frac{y-\theta \cdot}{t}\right)\right| \frac{|\cdot|}{t} \chi_{B_{j}}\right\|_{L^{q^{\prime} \cdot(\cdot)\left(\mathbb{R}^{n}\right)}} \\
& \leq C\left\|a_{j}\right\|_{L^{q(\cdot)}\left(\mathbb{R}^{n}\right)}\left\|\frac{|\cdot| \chi_{B_{j}}}{(|x-y|+|y-\theta \cdot|)^{n+1}}\right\|_{L^{q^{\prime}(\cdot)}\left(\mathbb{R}^{n}\right)} \\
& \leq C 2^{-j \alpha} \frac{1}{|x|^{n+1}}\left\||\cdot| \chi_{B_{j}}\right\|_{L^{q^{\prime}(\cdot)\left(\mathbb{R}^{n}\right)}} \leq C 2^{-j \alpha+j} \\
& \cdot \frac{1}{|x|^{n+1}}\left\|\chi_{B_{j}}\right\|_{L^{q^{\prime}(\cdot)}\left(\mathbb{R}^{n}\right)} \text {, }
\end{aligned}
$$

where $0 \leq \theta \leq 1$.

For $J_{12}$, by Lemma 15 we have

$J_{12}$

$$
\begin{aligned}
& \leq t^{-n} \int_{|z|>2^{j+1}}\left|K_{b^{\prime}} * a_{j}(z)\right|\left|\phi\left(\frac{y-z}{t}\right)-\phi\left(\frac{y}{t}\right)\right| d z \\
& \quad+t^{-n} \int_{|z|>2^{j+1}}\left|h * a_{j}(z)\right|\left|\phi\left(\frac{y-z}{t}\right)-\phi\left(\frac{y}{t}\right)\right| d z \\
& =: J_{13}+J_{14} .
\end{aligned}
$$

Noting that $2^{j} \geq 1$, then $|z|>2^{j+1} \geq 2$. Since $|z-w| \geq$ $|z|-|w|>2^{j} \geq 1$ for $|w| \leq 2^{j}$, we obtain

$$
\begin{aligned}
& \left|K_{b^{\prime}} * a_{j}(z)\right| \\
& \quad=\left|\int_{B_{j}} \frac{e^{i \alpha_{b}|z-w|^{-b^{\prime}}}}{|z-w|^{n}} \chi(|z-w| \leq 1) a_{j}(w) d w\right|=0 .
\end{aligned}
$$

So we have $J_{13}=0$.
For $J_{14}$, by the pointwise estimate for $h * a_{j}(z)$ in the proof of Theorem 6, we obtain

$$
\begin{aligned}
J_{14} & \leq C\left\|a_{j}\right\|_{L^{q(\cdot)}\left(\mathbb{R}^{n}\right)}\left\|\chi_{j}\right\|_{L^{q^{\prime}(\cdot)\left(\mathbb{R}^{n}\right)}} t^{-n} \\
& \times \int_{|z|>2^{j+1}}\left|\phi\left(\frac{y-z}{t}\right)-\phi\left(\frac{y}{t}\right)\right| \\
& \cdot\left[\frac{1}{(1+|z|)^{n+1}}+\frac{\chi(|z| \leq 2)}{|z|^{n-\varepsilon}}\right] d z \\
& \leq C\left\|a_{j}\right\|_{L^{q(\cdot)}\left(\mathbb{R}^{n}\right)}\left\|\chi_{j}\right\|_{L^{q^{\prime}(\cdot)\left(\mathbb{R}^{n}\right)}} \\
& \cdot t^{-n} \int_{|x| / 2>|z|>2^{j+1}}\left|\phi\left(\frac{y-z}{t}\right)-\phi\left(\frac{y}{t}\right)\right| \\
& \cdot \frac{1}{(1+|z|)^{n+1}} d z+C\left\|a_{j}\right\|_{L^{q(\cdot)}\left(\mathbb{R}^{n}\right)}\left\|\chi_{j}\right\|_{L^{q^{\prime}(\cdot)\left(\mathbb{R}^{n}\right)}} \\
& \cdot t^{-n} \int_{|z| \geq|x| / 2}\left|\phi\left(\frac{y-z}{t}\right)-\phi\left(\frac{y}{t}\right)\right| \frac{1}{(1+|z|)^{n+1}} d z \\
= & J_{15}+J_{16} \cdot
\end{aligned}
$$

Using the mean value theorem, we get

$J_{15}$

$$
\begin{aligned}
& \leq C\left\|a_{j}\right\|_{L^{q(\cdot)}\left(\mathbb{R}^{n}\right)}\left\|\chi_{j}\right\|_{L^{q^{\prime}(\cdot)\left(\mathbb{R}^{n}\right)}} \int_{|x| / 2>|z|>2^{j+1}} \sum_{|\beta|=1}\left|D^{\beta} \phi\left(\frac{y-\theta z}{t}\right)\right| \\
& \cdot\left|\frac{z}{t}\right| \frac{t^{-n}}{(1+|z|)^{n+1}} d z \\
& \leq C\left\|a_{j}\right\|_{L^{q \cdot(\cdot)\left(\mathbb{R}^{n}\right)}}\left\|\chi_{j}\right\|_{L^{q^{(\cdot)}\left(\mathbb{R}^{n}\right)}} \\
& \cdot \int_{|x| / 2>|z|>2^{j+1}} \frac{|z|}{(|x-y|+|y-\theta z|)^{n+1}} \frac{1}{(1+|z|)^{n+1}} d z \\
& \leq C\left\|a_{j}\right\|_{L^{q(\cdot)}\left(\mathbb{R}^{n}\right)}\left\|\chi_{j}\right\|_{L^{q^{(\cdot)}\left(\mathbb{R}^{n}\right)}} \frac{1}{|x|^{n+1}} \\
& \cdot \int_{|x| / 2>|z|>2^{j+1}} \frac{|z|}{(1+|z|)^{n+1}} d z \\
& \leq C\left\|a_{j}\right\|_{L^{q(\cdot)}\left(\mathbb{R}^{n}\right)}\left\|\chi_{j}\right\|_{L^{q^{\prime}(\cdot)}\left(\mathbb{R}^{n}\right)} \frac{\ln |x|}{|x|^{n+1}},
\end{aligned}
$$

where $0 \leq \theta \leq 1$.

For $J_{16}$, noting that $\phi \in \mathscr{K}_{m}$, we get

$$
\begin{aligned}
& J_{16} \leq C\left\|a_{j}\right\|_{L^{q(\cdot)}\left(\mathbb{R}^{n}\right)}\left\|\chi_{j}\right\|_{L^{q^{(\cdot)}\left(\mathbb{R}^{n}\right)}} \\
& \cdot t^{-n} \int_{|z| \geq|x| / 2}\left(\left|\phi\left(\frac{y-z}{t}\right)\right|+\left|\phi\left(\frac{y}{t}\right)\right|\right) \\
& \cdot \frac{1}{(1+|z|)^{n+1}} d z \leq C\left\|a_{j}\right\|_{L^{q(\cdot)}\left(\mathbb{R}^{n}\right)}\left\|\chi_{j}\right\|_{L^{q^{(\cdot)}\left(\mathbb{R}^{n}\right)}}
\end{aligned}
$$




$$
\begin{aligned}
& \cdot\left(\frac{1}{|x|^{n+1}}+\frac{t^{-n}}{(1+|y| /|t|)^{n}} \int_{|z| \geq|x| / 2} \frac{1}{(1+|z|)^{n+1}} d z\right) \\
& \leq C\left\|a_{j}\right\|_{L^{q^{(\cdot)}\left(\mathbb{R}^{n}\right)}}\left\|\chi_{j}\right\|_{L^{q^{\prime}(\cdot)\left(\mathbb{R}^{n}\right)}}\left(\frac{1}{|x|^{n+1}}\right. \\
& \left.+\frac{1}{|x|(|x-y|+|y|)^{n}}\right) \leq C\left\|a_{j}\right\|_{L^{q(\cdot)}\left(\mathbb{R}^{n}\right)}\left\|\chi_{j}\right\|_{L^{q^{\prime}(\cdot)\left(\mathbb{R}^{n}\right)}} \\
& \cdot \frac{1}{|x|^{n+1}} .
\end{aligned}
$$

Thus, for $|x|>2^{j+2}$, we get

$$
\begin{aligned}
& \left|G_{N}\left(T_{b} a_{j}\right)(x)\right| \\
& \quad \leq C\left\|a_{j}\right\|_{L^{q(\cdot)}\left(\mathbb{R}^{n}\right)}\left\|\chi_{B_{j}}\right\|_{L^{q^{\prime}(\cdot)}\left(\mathbb{R}^{n}\right)}\left(2^{j}+\ln |x|\right) \frac{1}{|x|^{n+1}} .
\end{aligned}
$$

So by using $0<p \leq 1, \alpha \leq 1+n \delta_{2}$, and Lemmas 9 and 10 we have

$$
\begin{aligned}
& J_{1}=\sum_{k=0}^{\infty} 2^{k \alpha p}\left(\sum_{j=0}^{k-1}\left|\lambda_{j}\right|\left\|G_{N}\left(T_{b} a_{j}\right) \chi_{k}\right\|_{L^{q(\cdot)}\left(\mathbb{R}^{n}\right)}\right)^{p} \\
& \leq C \sum_{k=0}^{\infty}\left(\sum_{j=0}^{k-1}\left|\lambda_{j}\right| 2^{k \alpha}\left\|a_{j}\right\|_{L^{q(\cdot)}\left(\mathbb{R}^{n}\right)}\left\|\chi_{B_{j}}\right\|_{L^{q^{\prime}(\cdot)}\left(\mathbb{R}^{n}\right)} 2^{j}\right. \\
& \left.\frac{1}{2^{k(n+1)}}\left\|\chi_{B_{k}}\right\|_{L^{q(\cdot)}\left(\mathbb{R}^{n}\right)}\right)^{p} \\
& +C \sum_{k=0}^{\infty}\left(\sum_{j=0}^{k-1}\left|\lambda_{j}\right| 2^{k \alpha}\left\|a_{j}\right\|_{L^{q(\cdot)}\left(\mathbb{R}^{n}\right)}\left\|\chi_{B_{j}}\right\|_{L^{q^{\prime} \cdot(\cdot)}\left(\mathbb{R}^{n}\right)} \ln 2^{k}\right. \\
& \left.\frac{1}{2^{k(n+1)}}\left\|\chi_{B_{k}}\right\|_{L^{q(\cdot)}\left(\mathbb{R}^{n}\right)}\right)^{p} \\
& \leq C \sum_{k=0}^{\infty}\left(\sum_{j=0}^{k-1}\left|\lambda_{j}\right| 2^{(j-k)\left(1+n \delta_{2}-\alpha\right)}\right)^{p} \\
& +C \sum_{k=0}^{\infty}\left(\sum_{j=0}^{k-1}\left|\lambda_{j}\right| 2^{j\left(n \delta_{2}-\alpha\right)} \frac{k}{2^{k\left(1+n \delta_{2}-\alpha\right)}}\right)^{p} \\
& \leq C \sum_{j=0}^{\infty}\left|\lambda_{j}\right|^{p} \sum_{k=j+1}^{\infty} 2^{(j-k)\left(1+n \delta_{2}-\alpha\right) p}+C \sum_{j=0}^{\infty}\left|\lambda_{j}\right|^{p} \\
& \cdot 2^{j\left(n \delta_{2}-\alpha\right) p} \sum_{k=j+1}^{\infty} \frac{k^{p}}{2^{k\left(1+n \delta_{2}-\alpha\right) p}} \leq C \sum_{j=0}^{\infty}\left|\lambda_{j}\right|^{p} \\
& +C \sum_{j=0}^{\infty}\left|\lambda_{j}\right|^{p} 2^{j\left(n \delta_{2}-\alpha\right) p} \frac{j^{p}}{2^{j\left(1+n \delta_{2}-\alpha\right) p}} \leq C \sum_{j=0}^{\infty}\left|\lambda_{j}\right|^{p} \\
& \leq C\|f\|_{H K_{q(\cdot)}^{\alpha, p}\left(\mathbb{R}^{n}\right)} .
\end{aligned}
$$

Therefore, by (67), (68), and (77) we complete the proof of Theorem 7 .

\section{Data Availability}

No data were used to support this study.

\section{Conflicts of Interest}

The authors declare that there are no conflicts of interest regarding the publication of this paper.

\section{Acknowledgments}

This work was supported by Shandong Provincial Natural Science Foundation, China (Grant No. ZR2017MA041), Project of Shandong Province Higher Educational Science and Technology Program (Grant No. J18KA225), China Postdoctoral Science Foundation (Grant No. 2016M601105), and National Natural Science Foundation of China (Grant Nos. 11761026, 11471309, and 11561062).

\section{References}

[1] O. Kováčik and J. Rákosník, "On spaces $\mathrm{L}^{p(x)}$ and $\mathrm{W}^{k, p(x), "}$ Czechoslovak Mathematical Journal, vol. 41, no. 4, pp. 592-618, 1991.

[2] H. Wang and Z. Liu, "The Herz-type Hardy spaces with variable exponent and their applications," Taiwanese Journal of Mathematics, vol. 16, no. 4, pp. 1363-1389, 2012.

[3] H. Wang and Z. Liu, "Some characterizations of Herz-type Hardy spaces with variable exponent," Annals of Functional Analysis, vol. 6, no. 2, pp. 224-233, 2015.

[4] C. Capone, D. Cruz-Uribe, and A. Fiorenza, "The fractional maximal operator and fractional integrals on variable $L^{p}$ spaces," Revista Matemática Iberoamericana, vol. 23, no. 3, pp. 743-770, 2007.

[5] D. Cruz-Uribe, A. Fiorenza, J. M. Martell, and C. Pérez, "The boundedness of classical operators on variable $L^{p}$ spaces," Annales Academice Scientiarum Fennico. Mathematica, vol. 31, no. 1, pp. 239-264, 2006.

[6] J. Tan and Z. Liu, "Some boundedness of homogeneous fractional integrals on variable exponent function spaces," Acta Mathematica Sinica. Chinese Series, vol. 58, no. 2, pp. 309-320, 2015.

[7] H. Wang, "Commutators of singular integral operator on Herztype Hardy spaces with variable exponent," Journal of the Korean Mathematical Society, vol. 54, no. 3, pp. 713-732, 2017.

[8] D. Cruz-Uribe, A. Fiorenza, and C. J. Neugebauer, "The maximal function on variable $L^{p}$ spaces," Annales Academice Scientiarum Fennicee Mathematica, vol. 28, no. 1, pp. 223-238, 2003.

[9] M. Izuki, "Boundedness of sublinear operators on Herz spaces with variable exponent and application to wavelet characterization," Analysis Mathematica, vol. 36, no. 1, pp. 33-50, 2010.

[10] I. I. Hirschman, "On multiplier transformations," Duke Mathematical Journal, vol. 26, no. 2, pp. 221-242, 1959.

[11] S. Wainger, "Special trigonometric series in k-dimension," Memoirs of the American Mathematical Society, vol. 59, p. 102, 1965. 
[12] C. Fefferman and E. M. Stein, " $H^{p}$ spaces of several variables," Acta Mathematica, vol. 129, no. 1, pp. 137-193, 1972.

[13] S. Chanillo, "Weighted norm inequalities for strongly singular convolution operators," Transactions of the American Mathematical Society, vol. 281, no. 1, p. 77, 1984.

[14] X. Li and S. Lu, "Strongly singular convolution operators on the weighted Herz-type Hardy spaces," Acta Mathematica Sinica, vol. 14, no. 1, pp. 67-76, 1998.

[15] E. Nakai and Y. Sawano, "Hardy spaces with variable exponents and generalized Campanato spaces," Journal of Functional Analysis, vol. 262, no. 9, pp. 3665-3748, 2012.

[16] L. Diening, P. Harjulehto, P. Hästö, and M. Ružička, Lebesgue and Sobolev Spaces with Variable Exponents, vol. 2017 of Lecture Notes in Mathematics, Springer, Berlin, Germany, 2011. 


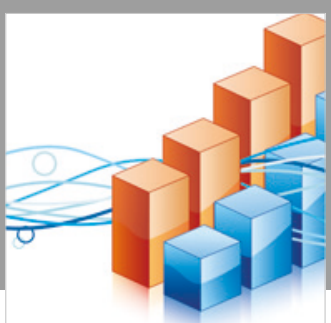

Advances in

Operations Research

\section{-n-m}
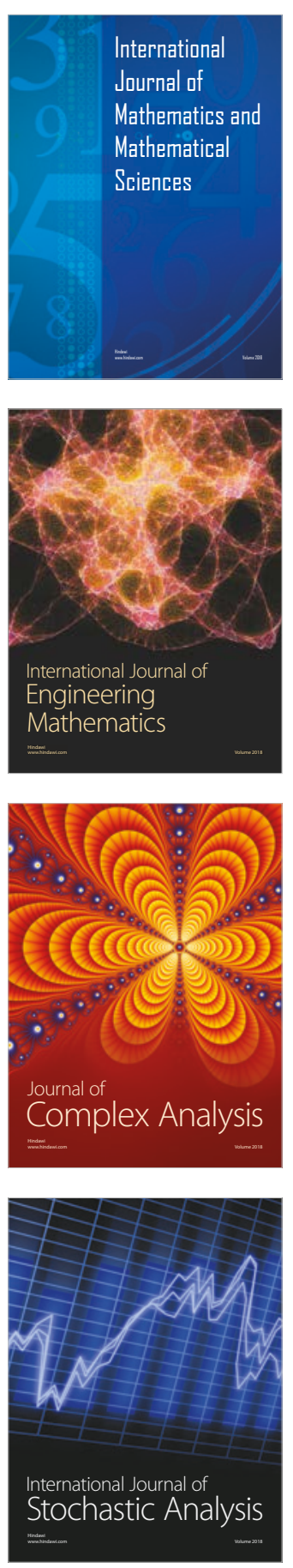
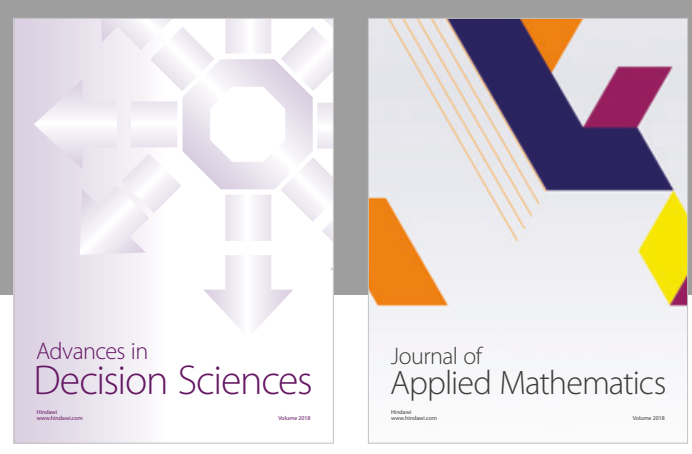

Journal of

Applied Mathematics
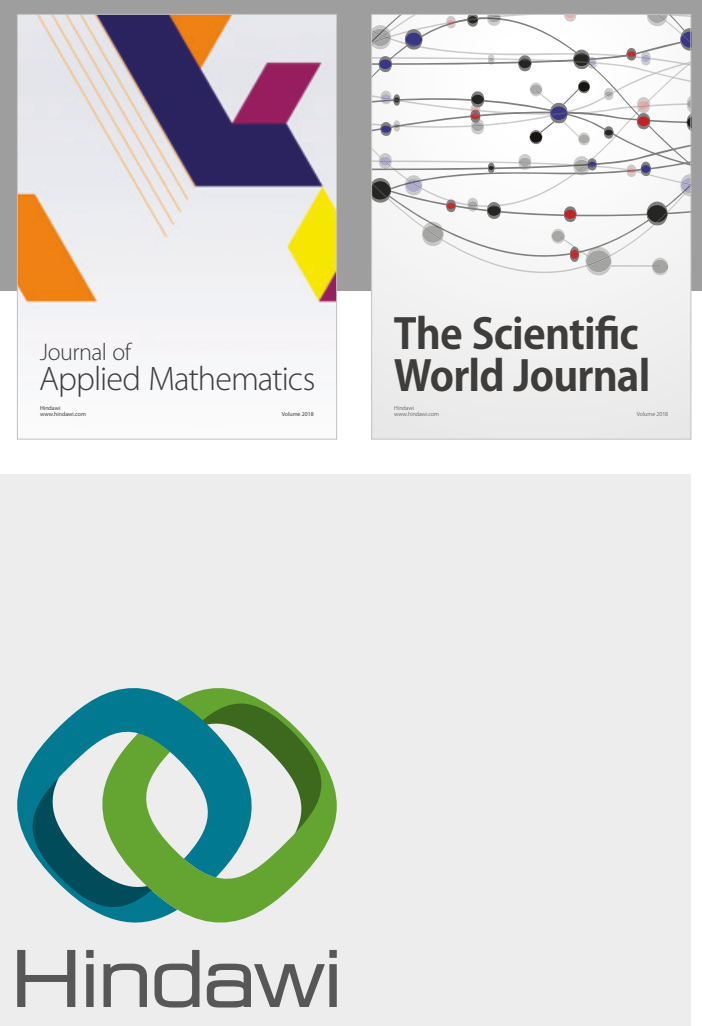

Submit your manuscripts at

www.hindawi.com

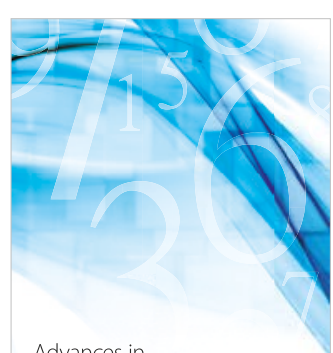

Advances in
Numerical Analysis
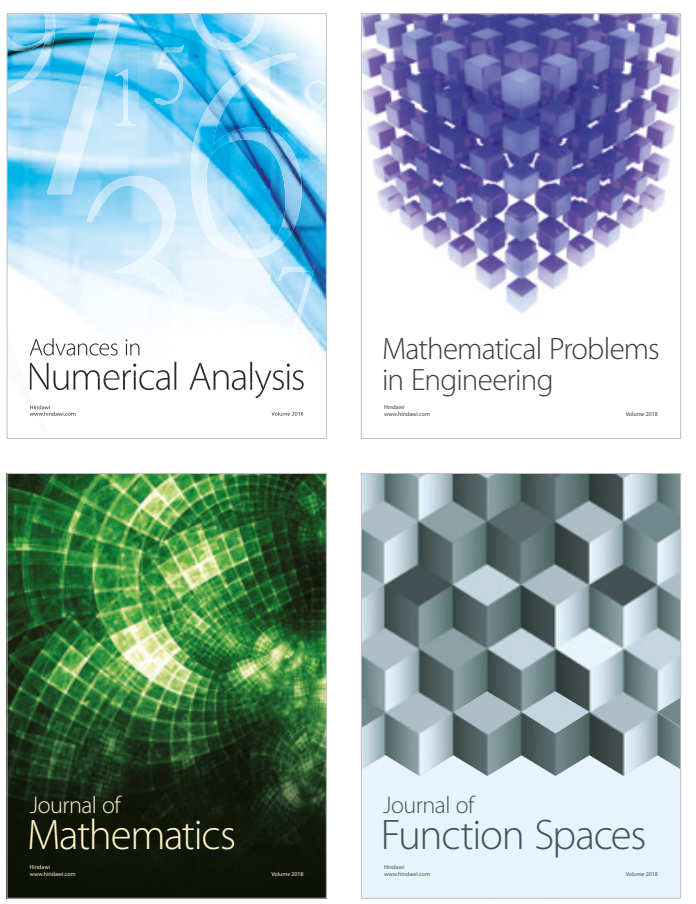

Mathematical Problems in Engineering

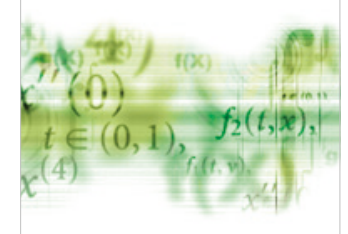

International Journal of

Differential Equations

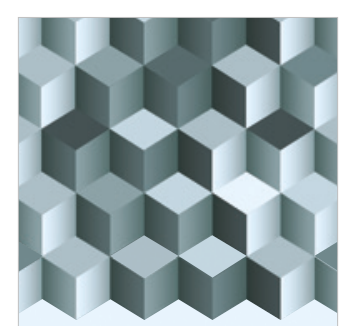

Journal of

Function Spaces

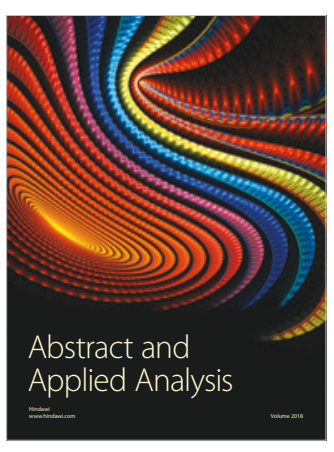

The Scientific

World Journal

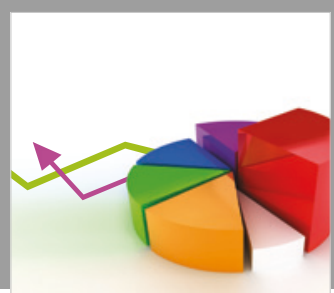

Journal of

Probability and Statistics
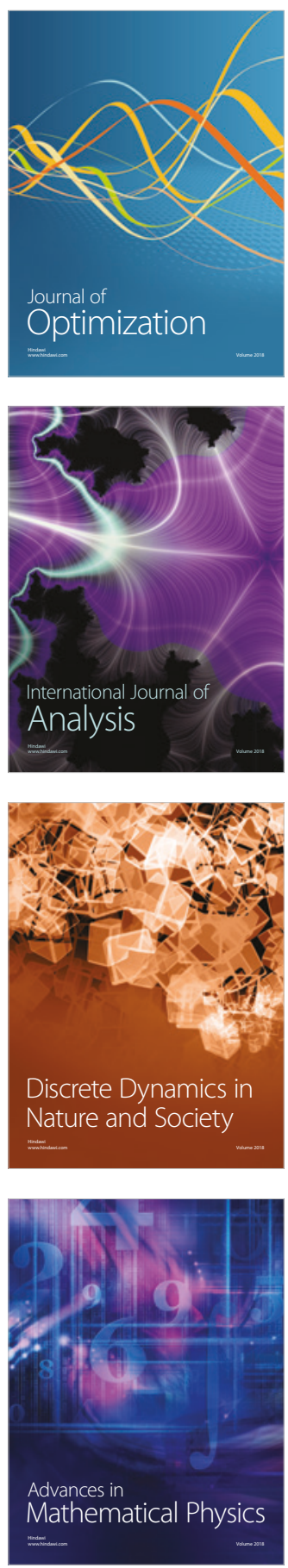